\title{
Holmium laser prostatectomy in a tertiary Italian center: A prospective cost analysis in comparison with bipolar TURP and open prostatectomy
}

\author{
Riccardo Schiavina ${ }^{1,2}$, Lorenzo Bianchi ${ }^{1,2}$, Marco Giampaoli ${ }^{3}$, Marco Borghesi ${ }^{1,2}$, \\ Hussam Dababneh $^{1,2}$, Francesco Chessa ${ }^{1,2}$, Cristian Pultrone ${ }^{1,2}$, Andrea Angiolini ${ }^{1}$, \\ Umberto Barbaresi $^{1}$, Matteo Cevenini ${ }^{1}$, Fabio Manferrari ${ }^{1,2}$, Alessandro Bertaccini ${ }^{1,2}$, \\ Angelo Porreca $^{3}$, Eugenio Brunocilla ${ }^{1,2}$ \\ ${ }^{1}$ Department of Urology, University of Bologna, Bologna, Italy; \\ 2 Department of Experimental, Diagnostic and Specialty Medicine (DIMES), Cardio-Nephro-Thoracic Sciences Doctorate, \\ University of Bologna, Bologna, Italy; \\ ${ }^{3}$ Department of Urology, Abano Terme Hospital, Padua, Italy.
}

\begin{abstract}
Summary Objective: To assess the economic impact of Holmium laser enucleation of prostate (HoLEP) in comparison with transurethral resection of prostate (TURP) and open prostatectomy (OP).

Methods: Between January 2017 and January 2018, we prospectively enrolled 151 men who underwent HoLEP, TURP or OP at tertiary Italian center, due to bladder outflow obstruction symptoms. Patients with prostate volume $\leq 70 \mathrm{cc}$ and those with prostate volume $>70 \mathrm{cc}$ were scheduled for TURP or HoLEP and OP or HoLEP, respectively.

Intraoperative and early post-operative functional outcomes were recorded up to 6 months follow up. Cost analysis was carried out considering direct costs (operating room [OR] utilization costs, nurse, surgeons and anesthesiologists' costs, OR disposable products costs and OR products sterilization costs), indirect costs (hospital stay costs and diagnostics costs) and global costs as sum of both direct and indirect plus general costs related to hospitalization. Cost analysis was performed comparing patients referred to TURP and HoLEP with prostate volume $\leq 70 \mathrm{cc}$ and men underwent $\mathrm{OP}$ and HoLEP with prostate volume $>70 \mathrm{cc}$ respectively.

Results: Overall, 53 (35.1\%), 51 (33.7\%) and 47 (31.1\%) were scheduled to HoLEP, TURP and OP, respectively. Both TURP, HoLEP and OP proved to effectively improve urinary symptoms related to BPE. Considering patients with prostate volume $\leq 70 \mathrm{cc}$, median global cost of HoLEP was similar to median global cost of TURP (2151.69 € vs. $2185.61 €$, respectively; $p=0.61$ ). Considering patients with prostate volume $>70 \mathrm{cc}$, median global cost of HoLEP was found to be significantly lower than median global cost of $O P$ (2174.15€ vs. $4064.97 €$, respectively; $p \leq 0.001$ ). Conclusions: Global costs of HoLEP are comparable to those of TURP, offering a cost saving of only $11.4 €$ in favor of HoLEP. Conversely, HoLEP proved to be a strong competitor of OP because of significant global cost sparing amounting to $1890.82 €$ in favor of HoLEP.
\end{abstract}

KEY WORDS: HoLEP; Xost analysis; TURP; Open prostatectomy; Prostatic enlargement.

Submitted 15 May 2019; Accepted 15 June 2019

\section{INTRODUCTION}

Benign prostatic enlargement (BPE) is one of the most common age-related medical disease in men, with troublesome impact on quality of life and a non-negligible social burden (1-3). The wide panorama of medical treatments developed over years has increasingly reduced the proportion of patients scheduled to surgical treatments (4). As a consequence, the economic impact of lower urinary tract symptoms (LUTS) is relevant, with costs exceeding 3 billion $\$$ annually in US (5), 320 million $€$ for pharmacological treatment and 74.834 days off work every year in Italy (6), and this trend is increasing over time (7). Surgical and endoscopic techniques, such as transurethral resection of prostate (TURP) and open prostatectomy (OP) have been the standard of care for many years in patients with drug-refractory disease. However, the surgical management of BPE has been changed in the last decade while laser prostatectomy increased in popularity (5).

Although different laser techniques are available for surgical treatment of BPE, Holmium laser enucleation of prostate (HoLEP) has been the most rigorously studied (8) and has emerged as a viable minim-invasive option in patients with symptomatic BPE regardless prostate volume (9). Several studies including randomized controlled trials demonstrated equivalent early- and long-term functional outcomes as compared to TURP $(10-13)$ and OP $(14,15)$, even in case of large prostate volume (16). Therefore, HoLEP is currently defined by International European Guidelines (17) as an effective alternative to TURP and OP, with several advantage of minim invasive approach including shorter catheterization time and hospital stay, reduced blood loss and lower blood transfusions $(10,11$, $14,15)$. Despite the initial non negligible costs, HoLEP could be less expensive by shortening the hospital stay and lowering the perioperative complications' rate if compared to TURP $(18,19)$ and OP $(2,16)$.

Since rigorous data on comparative costs of surgical treatments for BPE is limited in literature (20), we hereby aimed to assess the economic impact of HoLEP in com- 
parison with TURP and OP performed at single tertiary Italian center. Furthermore, we investigated and compared the surgical and early functional outcomes of the three surgical approaches.

\section{Material AND MEthods}

\section{Population}

Between January 2017 and January 2018, we prospectively enrolled 151 men who underwent surgical or endoscopic treatment for BPE at single tertiary Italian referral center (S. Orsola-Malpighi Hospital). Indications for surgical treatment consisted of persistent bladder outflow obstruction symptoms, International Prostatic Symptoms Score (IPSS) higher than 8, independent peak urinary flow rate $\left(Q_{\max }\right) \leq 15 \mathrm{ml} / \mathrm{s}$, or individuals non-responder to medical therapies including alfa blockers and $5 \alpha$-reductase inhibitors (5-ARI). The baseline assessment of BPE consist of digital rectal examination (DRE), transrectal ultrasound of prostate (TRUS) reporting the overall prostate volume and prostatic adenoma's volume, IPSS score, Quality of Life score $(\mathrm{QoL}), \mathrm{Q}_{\max }$, total PSA value and post void residual (PVR) measured with suprapubic ultrasound. Patients diagnosed with prostate cancer, those with history of previous prostatic or urethral surgery and with concomitant surgery needed (namely, bladder diverticulum excision and bladder stones removal) were excluded.

\section{Surgical techniques}

Patients with prostate volume $\leq 70 \mathrm{cc}$ and those with prostate volume $>70 \mathrm{cc}$ were scheduled for TURP or HoLEP and OP or HoLEP according to surgeon attitude and patient's preference, respectively.

TURP was carried out with a 26Fr continuous-flow Storz bipolar resectoscope, as previously described (21). All procedures were performed by 2 surgeons with more than ten years' experience with endoscopic surgery of both lower and upper urinary collecting system. A 22 Fr three-way catheter was positioned at the end of the procedure with continuous irrigation. HoLEP was performed by using Lumenis Versa Pulse ${ }^{\circledR}$ Holmium laser at $2.0 \mathrm{~J}$ and 50 pulses per second with a maximum average power of $100 \mathrm{~W}$ and 26Fr continuous-flow Storz laser resectoscope. Laser energy was delivered with a 550- $\mathrm{m}$ fiber. The enucleation of prostatic adenoma was performed according to Gilling's technique (22). The enucleated prostatic lobes were removed by using Lumenis VersaCut ${ }^{\mathrm{TM}}$ Morcellator System. All procedures were performed by a single surgeon at the end of learning curve with three years' experience with HoLEP technique and more than 200 procedures performed. A 22 Fr three-way catheter was positioned at the end of the procedure with continuous irrigation. OP was performed though trans-vesical approach as previous described (23). All procedures were performed by 4 surgeons with more than ten years' experience within OP techniques. A suprapubic drain and a 24Fr three-way catheter were positioned; the catheter was inflated in the prostatic fossa with a continuous irrigation.

\section{Collected data}

Each patient had complete preoperative data including
IPSS and QoL scores, $\mathrm{Q}_{\max }$ and PSA values, prostate volume and PVR at TRUS and suprapubic ultrasound, respectively. Recorded intraoperative data were as follows: surgical time, anesthesia time, total operating room (OR) usage time and removed tissue weight. Moreover, we measured early post-operative outcomes: Hemoglobin ( $\mathrm{Hg}$ ) loss at 24 hours after surgery, catheterization time, hospital stay and early complications including re-catheterization, clot irrigation, transfusion and urinary tract infection according to Clavien-Dindo classification (24).

After discharge, patients were scheduled to follow up examination at 3,6 and 12 months including IPSS and QoL scores, PVR and $\mathrm{Q}_{\max }$. Moreover, urge and stress incontinence rates were recorded after discharge, at 3,6 and 12 months follow up. Urinary incontinence was defined as usage of $\geq 1$ PAD per day. At time of analyses, all patients had complete follow up data up to 6 months after surgery. Cost analysis was carried out thought Delta analysis considering direct costs related to surgical procedure, indirect costs related to post-surgical hospitalizations and global costs as sum of both direct and indirect plus general costs related to hospitalization. Those data were obtained with the collaboration of the Finance Department with our center.

Direct costs consist of OR utilization costs (2,01 €/minute) plus Nurse cost $(0,5 € /$ minute, considering 3 nurses in $\mathrm{OP}$ and 2 nurses in HoLEP and TURP), O.R. personnel costs including surgeons (1,33 €/minute, considering 2 surgeons in OP and 1 Surgeon in HoLEP and TURP) and anesthesiologists (1,33 €/minute), O.R. disposable products costs and O.R. products sterilization costs. Indirect costs included hospital stay costs, diagnostics costs and costs of complications (including additional drugs, transfusions and medications). General costs included several costs of different services for each patient calculated by the hospital's administration and related to the hospital stay (including insurance, water and electricity).

\section{Data analysis and statistical assessments}

First, preoperative patients' characteristics and mildterm ( $\leq 6$ months) postoperative data were compared between patients referred to the three surgical approaches (namely, OP, TURP and HoLEP): Anova test was used to compare continuous variables between the three groups. Second, postoperative stress and urge incontinence rates were reported at discharge, at 3 and 6 months after surgery and were compared between the three surgical techniques using chi-square test.

Third, intraoperative and early postoperative data as well as intraoperative surgical times and surgical costs were analyzed comparing patients referred to HoLEP and TURP and those scheduled to HoLEP and OP, respectively. Since surgical indication to TURP or OP was respectively carried out in case of prostate volume $\leq 70 \mathrm{cc}$ and $>70 \mathrm{cc}$ at preoperative TRUS, while the indication to HoLEP was carried out regardless prostate volume, individuals referred to HoLEP with prostate volume $\leq 70 \mathrm{cc}(\mathrm{HoLEP} \leq 70 \mathrm{cc})$ and those with prostate volume $>70 \mathrm{cc}(\mathrm{HoLEP}>70 \mathrm{cc})$ were compared with men underwent TURP and OP, respectively, in order to assess whenever HoLEP technique could represent a direct competitor of both standard surgical procedures according to prostate volume. 
Statistical analysis was conducted with IBM SPSS 21 with a 2 -sided significance level set at $\mathrm{P}<0.05$.

The local institutional ethical committee approved the study (approval code STUD-OF by the S. Orsola-Malpighi Hospital, IRB September 11, 2012).

\section{RESULTS}

\section{Preoperative data}

Overall, 151 patients were prospectively enrolled. Of them, 53 (35.1\%), 51 (33.7\%) and 47 (31.1\%) were scheduled to HoLEP, TURP and OP, respectively. Among patients submitted to HoLEP, 27 (50.1\%) individuals had a preoperative prostatic volume $\leq$ 70 cc, while 26 (49.9\%) had a preoperative prostatic volume $>70 \mathrm{cc}$. Between patients referred to OP, TURP and HoLEP, the preoperative clinical characteristics including age, IPSS and QoL scores, $\mathrm{Q}_{\max }$ and PVR were found to be similar between the three

Table 1.
Preoperative patients' characteristics according to the surgical techniques (namely, OP, TURP and HoLEP).

\begin{tabular}{|c|c|c|c|c|}
\hline & OP & TURP & HOLEP & $P$ value \\
\hline Number of patients (\%) & $47(31.1)$ & $51(33.7)$ & $53(35.1)$ & - \\
\hline \multicolumn{5}{|l|}{ Age (years) } \\
\hline Mean \pm SD (range) & $71.1 \pm 7.3(56-85)$ & $69.0 \pm 9.7(46-86)$ & $70.2 \pm 6.8(51-84)$ & 0.4 \\
\hline \multicolumn{5}{|l|}{ Prostate Volume (cc) } \\
\hline Mean \pm SD (range) & $109.8 \pm 45.8(75-280)$ & $43.3 \pm 13.1(27-70)$ & $75.4 \pm 25.6(32-140)$ & $<0.001$ \\
\hline \multicolumn{5}{|l|}{$Q_{\max }(\mathrm{mL} / \mathrm{sec})$} \\
\hline Mean \pm SD (range) & $8.5 \pm 4.4(2-15)$ & $9.5 \pm 4.9(4-15)$ & $9.1 \pm 3.6(4-15)$ & 0.9 \\
\hline \multicolumn{5}{|l|}{ IPSS } \\
\hline Mean \pm SD (range) & $15.6 \pm 8.2(8-33)$ & $19.6 \pm 7.7(8-34)$ & $17.2 \pm 7.2(8-30)$ & 0.07 \\
\hline \multicolumn{5}{|l|}{$\overline{Q 0 L}$} \\
\hline Mean \pm SD (range) & $3.6 \pm 1.5(1-6)$ & $4.1 \pm 1.3(1-6)$ & $3.8 \pm 1.4(1-6)$ & 0.2 \\
\hline \multicolumn{5}{|l|}{ PVR (cc) } \\
\hline Mean \pm SD (range) & $96.7 \pm 65.3(10-300)$ & $87.1 \pm 55.8(10-250)$ & $88.7 \pm 60.9(10-220)$ & 0.9 \\
\hline \multicolumn{5}{|l|}{ PSA (ng/mL) } \\
\hline Mean \pm SD (range) & $6.73 \pm 3.29(1.5-15.8)$ & $2.55 \pm 2.34(0.5-11.0)$ & $3.27 \pm 2.46(0.6-12.0)$ & $<0.001$ \\
\hline
\end{tabular}

\section{Table 2.}

Preoperative, surgical and early post-operative outcomes according to the surgical techniques (namely, TURP vs. HoLEP with preoperative prostatic volume $\leq 70 \mathrm{cc}$ and $\mathrm{OP}$ vs HoLEP with preoperative prostatic volume $>70 \mathrm{cc}$ ).

\begin{tabular}{|c|c|c|c|c|c|c|}
\hline & TURP & HoLEP $\leq 70$ cc & $P$ value & $\mathrm{OP}$ & HoLEP > 70 cc & $P$ value \\
\hline Number of patients (\%) & $51(33.7)$ & $27(17.9)$ & - & $47(31.1)$ & $26(17.2)$ & - \\
\hline \multicolumn{7}{|c|}{ PREOPERATIVE CHARACTERISTICS } \\
\hline \multicolumn{7}{|l|}{ Age (years) } \\
\hline Mean \pm SD (range) & $69.0 \pm 9.7(46-86)$ & $70.9 \pm 6.7(51-83)$ & 0.3 & $71.1 \pm 7.3(56-85)$ & $69.5 \pm 7.03(57-84)$ & 0.6 \\
\hline \multicolumn{7}{|l|}{ Prostate volume (cc) } \\
\hline Mean \pm SD (range) & $43.3 \pm 13.1(27-70)$ & $47.8 \pm 8.3(32-70)$ & 0.06 & $109.8 \pm 45.8(75-280)$ & $96.8 \pm 18.8(75-140)$ & 0.06 \\
\hline \multicolumn{7}{|l|}{$\overline{Q_{\max }}(\mathrm{mL} / \mathrm{sec})$} \\
\hline Mean \pm SD (range) & $9.5 \pm 4.9(4-15)$ & $8.7 \pm 2.7(5-15)$ & 0.6 & $8.5 \pm 4.4(2-15)$ & $9.7 \pm 4.5(3.7-15)$ & 0.6 \\
\hline \multicolumn{7}{|l|}{ IPSS } \\
\hline Mean \pm SD (range) & $19.6 \pm 7.7(3-34)$ & $16.1 \pm 6.5(3-27)$ & 0.06 & $15.6 \pm 8.2(3-33)$ & $18.3 \pm 7.8(3-30)$ & 0.06 \\
\hline \multicolumn{7}{|l|}{$\overline{\text { QOL }}$} \\
\hline Mean \pm SD (range) & $4.1 \pm 1.3(1-5)$ & $3.8 \pm 1.2(1-5)$ & 0.2 & $3.6 \pm 1.5(1-5)$ & $4.3 \pm 1.5(1-5)$ & 0.2 \\
\hline \multicolumn{7}{|l|}{ PVR (cc) } \\
\hline Mean \pm SD (range) & $87.1 \pm 55.8(10-250)$ & $95.4 \pm 72.3(15-220)$ & 0.3 & $96.7 \pm 65.3(10-300)$ & $96.6 \pm 62.8(10-220)$ & 0.3 \\
\hline \multicolumn{7}{|l|}{$\overline{\mathrm{PSA}}(\mathrm{ng} / \mathrm{mL})$} \\
\hline Mean \pm SD (range) & $2.55 \pm 2.34(0.5-11.0)$ & $2.50 \pm 1.80(0.6-7.6)$ & 0.9 & $6.73 \pm 3.29(1.5-15.8)$ & $4.00 \pm 2.80(1.1-12.0)$ & 0.9 \\
\hline \multicolumn{7}{|c|}{ INTRAOPERATIVE DATA } \\
\hline \multicolumn{7}{|l|}{ Removed Tissue Weight (gr) } \\
\hline Mean \pm SD (range) & $21.6 \pm 11.1(5-50)$ & $26.4 \pm 12.2(20-55)$ & 0.09 & $62.2 \pm 32.7(30-180)$ & $50.2 \pm 23.2(31-90)$ & 0.09 \\
\hline \multicolumn{7}{|c|}{$\begin{array}{l}\text { Intra-perioperative complications } \\
\text { according to Clavien-Dindo classification (\%) }\end{array}$} \\
\hline Overall & $5(10)$ & $1(4)$ & 0.3 & $11(23)$ & $2(8)$ & $0.04^{*}$ \\
\hline Grade 1 & $3(6)$ & $0(0)$ & & $3(6)$ & $1(4)$ & \\
\hline Grade 2 & $2(4)$ & $1(4)$ & & $6(13)$ & $0(0)$ & \\
\hline Grade 3 & $0(0)$ & $0(0)$ & & $2(4)$ & $1(4)$ & \\
\hline \multicolumn{7}{|c|}{ EARLY POST-OPERATIVE OUTCOMES } \\
\hline \multicolumn{7}{|l|}{ Hb loss at 24 hours (g/dL) } \\
\hline Mean \pm SD (range) & $1.1 \pm 0.7(0-3.9)$ & $2.1 \pm 3.4(0.3-3.6)$ & 0.1 & $2.1 \pm 1.4(0-6.2)$ & $1.9 \pm 1.2(0-5.2)$ & 0.2 \\
\hline \multicolumn{7}{|l|}{ Catheterization time (hr) } \\
\hline Mean \pm SD (range) & $74.4 \pm 21.4(48-144)$ & $57.2 \pm 43.9(20-183)$ & $0.003^{*}$ & $146.9 \pm 55.6(60-448)$ & $68.1 \pm 53.8(20-200)$ & $<0.001^{*}$ \\
\hline \multicolumn{7}{|l|}{ Hospital stay (hr) } \\
\hline Mean \pm SD (range) & $84.4 \pm 10.5(60-104)$ & $72.2 \pm 37.4(27-168)$ & $0.01 *$ & $184.2 \pm 78.7(84-554)$ & $83.9 \pm 42.3(47-192)$ & $<0.001^{*}$ \\
\hline
\end{tabular}

groups, except for prostate volume that was significantly higher in men treated with OP and HoLEP as com- 
pared to those referred to TURP (Table $1 ; \mathrm{p}<0.001$ ). However, no significant differences were found between men referred to TURP and those underwent HoLEP with prostate volume $\leq 70 \mathrm{cc}$ and between patients referred to OP as compared with those submitted to HoLEP with prostate volume $>70$ $\mathrm{cc}$, concerning preoperative characteristics (Table 2).

\section{Perioperative data}

Concerning mean removed tissue weight, we found no significant difference between patients referred to TURP as compared with those submitted to HoLEP with prostate volume $\leq 70 \mathrm{cc}$ and between patients referred to OP as compared with those submitted to HoLEP with prostate volume $>70 \mathrm{cc}$ (Table 2). Men in TURP group and those in $\mathrm{OP}$ group revealed higher catheterization and hospital stay times as referred to individuals scheduled to HoLEP regardless prostate volume (all $\mathrm{p} \leq 0.01$; Table 2). In men with prostate volume $\leq 70$ cc, both HoLEP and TURP revealed to be safety procedures with $4 \%$ and $10 \%$ overall complications, respectively $(p=0.3)$. On the contrary, in men with prostate volume $>70 \mathrm{cc}$, those referred to OP experienced higher rates and higher grade of complications as referred to those underwent to HoLEP $(\mathrm{p} \leq 0.04)$.

\section{Postoperative functional outcomes}

Both TURP, HoLEP and OP proved to effectively improve urinary symptoms related to BPE at short term follow up, since no significant differences were found in term of IPSS score, $\mathrm{Q}_{\max }$, QoL score and PVR at time of discharge, at 3 and 6 months after surgery, between the three groups (Table 3).

Patients in HoLEP group revealed higher stress incontinence rate at 3 months after surgery as compared to men in TURP and OP group, despite not significant difference ( $8 \%$ vs. $4 \%$ vs. $2 \%$; Figure 1; $\mathrm{p}=0.2)$; however, only $1(2 \%)$ patients referred to HoLEP and 1 (2\%) men underwent OP, experienced stress incontinence at 6 months follow up. Irritative symptoms were comparable between the three surgical approach and only 1 patient $(2 \%)$ had urge incontinence at 6 months after HoLEP (Figure 2).

Table 3. and HoLEP).

\section{Figure 1.}

Figure 2.
Short-term functional outcomes of the three surgical techniques (namely, OP, TURP

\begin{tabular}{|c|c|c|c|c|c|c|c|c|}
\hline & OP (47) & $\begin{array}{l}3 \text { months } \\
\text { TURP (51) }\end{array}$ & HoLEP (53) & $P$ value & $\begin{array}{l}6 \text { months } \\
\text { OP (47) }\end{array}$ & TURP (51) & HoLEP (53) & $P$ value \\
\hline IPSS & & & & & & & & \\
\hline Mean \pm SD & $7.6 \pm 4.2$ & $9.6 \pm 7.0$ & $9.5 \pm 5.8$ & 0.3 & $7 \pm 4.5$ & $8.57 \pm 5.7$ & $8.3 \pm 5.6$ & 0.5 \\
\hline 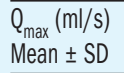 & $23.8 \pm 10.6$ & $21.5 \pm 8.5$ & $22.8 \pm 10.5$ & 0.7 & $23.2 \pm 10.8$ & $22.8 \pm 9.7$ & $22.7 \pm 9.6$ & 0.2 \\
\hline $\begin{array}{l}\text { QoL } \\
\text { Mean } \pm \text { SD }\end{array}$ & $1.2 \pm 1.3$ & $1.8 \pm 1.6$ & $1.5 \pm 1.5$ & 0.5 & $0.9 \pm 1.0$ & $1.4 \pm 1.1$ & $1.4 \pm 1.3$ & 0.2 \\
\hline $\begin{array}{l}\text { PVR (cc) } \\
\text { Mean } \pm \text { SD }\end{array}$ & $3.5 \pm 2.1$ & $10.9 \pm 22.8$ & $20.8 \pm 12.1$ & 0.4 & $4.1 \pm 2.5$ & $10.8 \pm 25.3$ & $24.5 \pm 16.6$ & 0.4 \\
\hline
\end{tabular}

\section{Intraoperative times and costs analyses}

O.R usage, anesthesiology and surgery time and disposable products' costs revealed to be significantly higher in patients referred to HoLEP $\leq 70 \mathrm{cc}$, as compared to those treated with TURP (all $\mathrm{p} \leq 0.001$ ). Accordingly, median direct costs of HoLEP in men with prostate volume $\leq 70$ cc were significantly higher as compared to median direct

Postoperative stress urinary incontinence rate according to the three surgical techniques (namely, OP, TURP and HoLEP) at discharge, 3 and 6 months follow up.

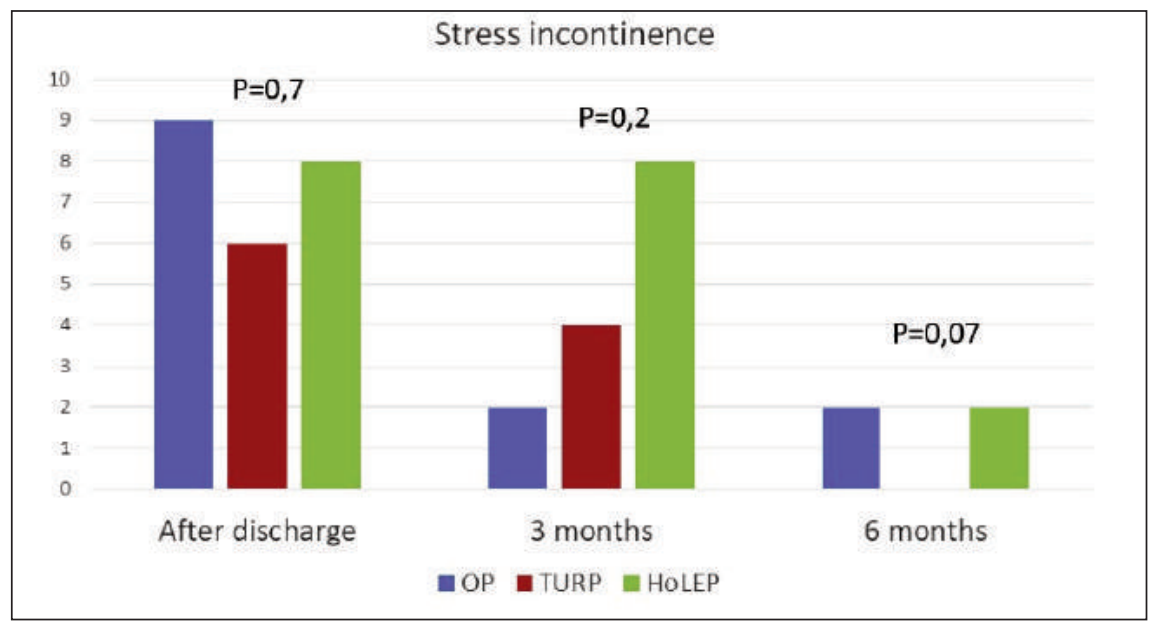

Postoperative urge urinary incontinence rate according to the three surgical techniques (namely, OP, TURP and HoLEP) at discharge, 3 and 6 months follow up.

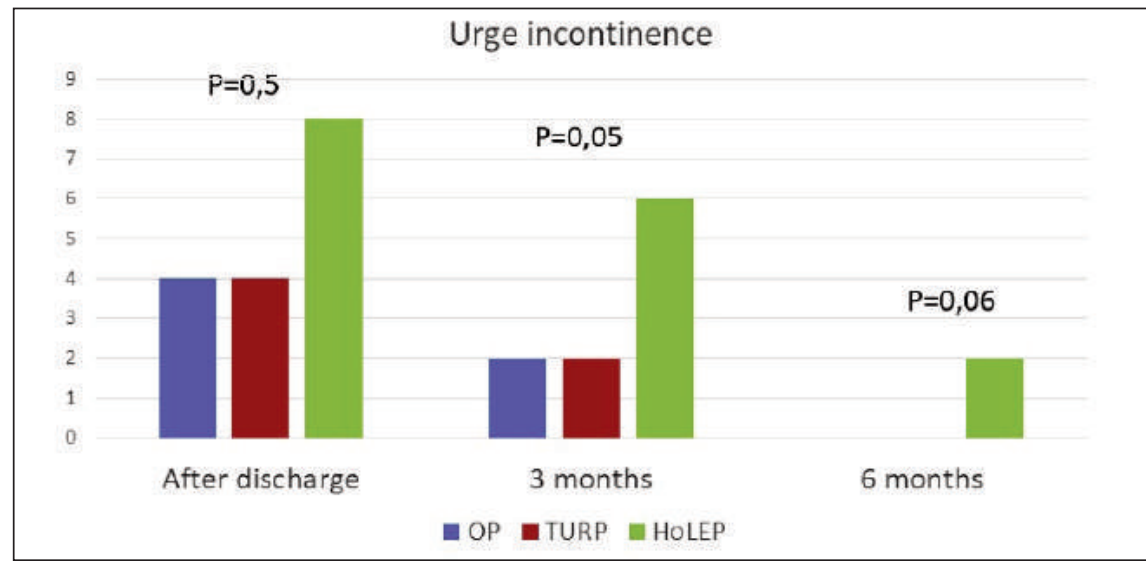


Table 4.

Cost analysis including direct, indirect and total costs according to surgical procedure (namely, TURP vs. HoLEP with preoperative prostatic volume $\leq 70 \mathrm{cc}$ and $\mathrm{OP}$ vs HoLEP with preoperative prostatic volume $>70 \mathrm{cc}$ ).

\begin{tabular}{|c|c|c|c|c|c|c|}
\hline & TURP & HoLEP $\leq 70 \mathrm{cc}$ & $P$ value & OP & HoLEP > $70 \mathrm{cc}$ & $P$ value \\
\hline Number of patients (\%) & $51(33.7)$ & $27(17.9)$ & $\cdot$ & 47 (31.1) & $26(17.2)$ & - \\
\hline \multicolumn{7}{|l|}{ 0.R. usage time (min) } \\
\hline Median (IQR) & $105(89.75-125)$ & $140(113.25-176)$ & $<0.001^{*}$ & $110(90-119)$ & $140(117-175)$ & $<0.001^{*}$ \\
\hline \multicolumn{7}{|l|}{ 0.R usage cost ( $(€)$} \\
\hline Median (IQQR) & $306.23(270.14-376.25)$ & $421.40(332.61-535.78)$ & $<0.001^{*}$ & $386.10(315.90-417.69)$ & $421.40(349.91-527.50)$ & $0.04^{*}$ \\
\hline \multicolumn{7}{|l|}{ Anesthesiology time (min) } \\
\hline Median (IQR) & 88 (74.5-111) & $123(103-161.5)$ & $<0.001^{*}$ & $93(80-110)$ & $117(100-160)$ & $<0.001 *$ \\
\hline \multicolumn{7}{|l|}{ Anestesiology cost (€) } \\
\hline Median (IQR) & $117.23(99.09-147.63)$ & 167.58 (129.01-214.13) & $<0.001^{*}$ & $123.69(106.40-146.30)$ & $154.94(133.00-215.13)$ & $<0.001 *$ \\
\hline \multicolumn{7}{|l|}{ Surgery time (min) } \\
\hline Median (IQR) & $63(47-85.25)$ & 96 (70.5-123.25) & $<0.001^{*}$ & $68(58-89)$ & $96(72-120)$ & $0.001^{*}$ \\
\hline \multicolumn{7}{|l|}{ Surgeon cost $(t)$} \\
\hline Median (IQR) & 84.78 (62.51-113.38) & $141.00(89.76-161.60)$ & $<0.001^{*}$ & $180.88(154.28-236.74)$ & $114.38(95.76-162.26)$ & $<0.001 *$ \\
\hline Disposable products $(€)$ & 34.80 & 134.04 & $<0.001^{*}$ & 34.8 & 134.04 & $<0.001 *$ \\
\hline$\overline{\text { Sterilization costs }(€)}$ & 103.86 & 103.86 & & 103.86 & 103.86 & 1.0 \\
\hline \multicolumn{7}{|l|}{ Direct costs $(€)$} \\
\hline Median (IQR) & $650.90(559.46-760.41)$ & 866.62 (717.94-1040.52) & $<0.001^{*}$ & 948.89 (813.15-1054.59) & 803.31 (723.30-1074.98) & 0.09 \\
\hline \multicolumn{7}{|l|}{ Indirect costs $(€)$} \\
\hline Median (IQR) & $1218.29(1214.36-1272.83)$ & 889.13 (797.77-1433.61) & $0.002 *$ & 2542.87 (2194.06-2885.68) & 867.20 (794.12-1203.39) & $<0.001 *$ \\
\hline \multicolumn{7}{|l|}{ Direct + Indirect costs $(€)$} \\
\hline Median (IQR) & 1868.19 (1771.48-2058.04) & 1772.33 (1418.25-2234.13) & 0.37 & 3507.33 (3205.78-3895.28) & 1905.60 (1509.39-2132.37) & $<0.001^{*}$ \\
\hline \multicolumn{7}{|l|}{ General costs (€) } \\
\hline Median (IQR) & $317.42(301.15-348.17)$ & $301.29(241.10-379.80)$ & 0.38 & $596.24(544.98-662.19)$ & $323.95(256.59-362.50)$ & $<0.001 *$ \\
\hline \multicolumn{7}{|l|}{ Total cost $(€)$} \\
\hline Median (IQR) & $2185.61(2072.64-2396.20)$ & 2151.69 (1735.79-2618.85) & 0.61 & 4064.97 (3636.36-4557.47) & 2174.15 (1765.23-2465.19) & $<0.001^{*}$ \\
\hline
\end{tabular}

costs of TURP (866.62 € vs. $650.90 €$, respectively; $\mathrm{p} \leq$ 0.001 ; Table 4). On the contrary, indirect costs were found to be inferior in HoLEP $\leq 70 \mathrm{cc}$ group as compared to TURP group (all p $\leq 0.002$ ), mainly due to lower hospitalization time. As a matter of fact, considering patients with prostate volume $\leq 70 \mathrm{cc}$, median global cost of HoLEP was similar to median global cost of TURP (2151.69 € vs. 2185.61 €, respectively; $\mathrm{p}=0.61$; Table 3). Despite significant difference in terms of O.R usage, anesthesiology and surgery time and disposable products' costs between patients referred to HoLEP > $70 \mathrm{cc}$ and OP in favor of OP (all $\mathrm{p}<0.001$ ), median direct costs revealed to be similar between OP and HoLEP in men with prostate volume > $70 \mathrm{cc}(948.89 €$ vs. $803.31 €$; $\mathrm{p}=0.09$; Table 4). This could be explained with higher surgeon costs in OP which is related to the involvement of two surgeons per procedure, although the surgical time is lower in OP as referred to HoLEP which is a single surgeon procedure. On the other side, indirect costs were found to be significantly lower in HoLEP > $70 \mathrm{cc}$ group as compared to OP group ( $p<0.001$ ), mainly due to lower hospitalization time and lower complications rates. Therefore, considering patients with prostate volume $>70 \mathrm{cc}$, median global cost of HoLEP was found to be significantly lower than median global cost of OP (2174.15 $€$ vs. $4064.97 €$, respectively; $\mathrm{p} \leq 0.001$; Table 4).

\section{Discussion}

Thanks to unquestionable efficiency, early and long terms functional outcomes and safety profile, TURP represents nowadays the gold standard (17), (25) for surgical treatment of patients affected by BPE. On the other hand, laser technology has been worldwide increasingly diffused as safe minim invasive surgical treatment for BPE. Among different laser adopted in urology, Holmium laser has been the most rigorously studied (8) and HoLEP has passes the test of the time. On the other side, OP has been the first choice of surgical treatment in men with a substantially enlarged prostate (namely, prostate volume $>80 \mathrm{cc}$ ) in the last 50 years, despite more invasive approach and higher operative morbidity. However, contrarily to TURP, the rate of open procedures varies among different countries and cultures, because of different national health systems, variable economic pressure and available resources. In fact, analysis of direct and indirect costs suggests that OP is the most expensive surgical procedure for BPE (23). Taken together these considerations support that OP is a technique of the past which would be progressively abandoned. Contrarily, HoLEP proved to be a safe alternative to TURP (10-13) and OP $(14,15)$ with equivalent early and long term functional outcomes $(10,11,14,15)$, that render HoLEP an attractive competitor of both standard techniques. Despite such benefits, two main drawbacks including a steep learning curve and the costs related to initial laser equipment (Holmium laser, a dedicated laser resectoscope sheath, fibers and morcellator) could have limited diffusion of this technique. Nevertheless, it has been postulated that HoLEP is a cost-sparing procedure since fibers can be reused multiple times and holmium laser can be used for several other urological procedures (26). Moreover, previous authors showed that HoLEP is more 
cost-effective as compared to TURP $(18,19)$ and OP $(2$, 16), giving a cost savings of $24.5 \%$ (18) and 9.6\% (2), respectively.

Our cost-analysis attempted to evaluate and compare the financial burden of different procedures for surgical treatment of BPE performed at single Italian institution, in order to define a future perspective concerning surgical management of patients with BPE. Indeed, several findings are noteworthy.

First, HoLEP proved to be as efficient as TURP and OP in term of removed tissue weight. Second, our study provides further evidence to support optimal functional outcomes of HoLEP at short follow up. Moreover, HoLEP confirmed to be a safe procedure, since similar intraoperative and perioperative complications were found as compared to TURP. Conversely, HoLEP proved to be a safer approach than $\mathrm{OP}$, considering lower complications rates compared to OP ( $8 \%$ vs. $23 \%$; Table 2). Precisely, no men in HoLEP $>70 \mathrm{cc}$ and 6 patients in OP group required blood transfusions; moreover, only 1 HoLEP $>70 \mathrm{cc}$ and $2 \mathrm{OP}$ needed re-intervention in order to achieve control of bleeding. Third, according to literature (25), patients submitted to HoLEP experienced faster recovery due to significantly lower catheterization time and hospital stay as compared to those underwent TURP and OP regardless prostate volume (Table 2).

Fourth, direct costs, including OR surgical setup, disposables, fibers and surgical staff costs (namely, unitary cost of surgeon, anesthesiology and operating room nurses) of HoLEP $\leq 70 \mathrm{cc}$ procedures, were found to be significantly higher as related to TURP's costs. A sub-analysis of direct costs shows as disposable costs and sterilization costs are similar between two techniques. Conversely, higher direct costs within HoLEP $\leq 70 \mathrm{cc}$ group, seems to be related mainly to increased operating room usage time, that leads to augment costs of surgeon, anesthesiologists and all staff involved. However, indirect costs found to be significantly lower in patients referred to HoLEP $\leq 70 \mathrm{cc}$ as compared to those treated with TURP, due to lower hospital stay. In fact, laser technology allows to spare almost 1 day of hospitalization, thanks to optimal hemostatic proprieties and earlier catheter removal. Therefore, global HoLEP's costs found to be comparable to global TURP's costs considering patients with similar prostate volume $(\leq 70 \mathrm{cc})$. This implies an overall cost saving of $11.4 €$ per procedure, in favor of HoLEP. Our findings differ from previous costeffectiveness analysis reported by Fraundorfer et al. (18) comparing Holmium laser prostatectomy (namely, Holmium laser resection of prostate) and TURP, by suggesting a net economic benefit of 24.5\% (651 New Zeland dollars) in favor of laser. However, the authors did not include in their analysis the medical salary costs (urologist and anesthesiologist), that could reduce the cost-saving difference between two techniques.

Fifth, our economic analysis shows that HoLEP could be an attractive competitor of OP. In fact, direct costs of HoLEP were found to be comparable to OP, considering patients with similar prostate volume (> $70 \mathrm{cc}$ ). Despite lower operating room usage time and related costs, in favor of OP, that would reduce surgeon cost, the median surgeon cost of OP (180.88 €) is significantly higher as compared with those of HoLEP (114.38 €) since it is influenced by the number of surgeons involved in each procedure (namely, two surgeons in OP and one surgeon in HoLEP). Indeed, the higher costs of disposable products in HoLEP group, mainly related to the costs of fibers, can be amortized during time, since a single fiber can be re-used at least 10 times. Moreover, the main aspect that renders HoLEP an attractive and preferable procedure as referred to OP, consists of lower hospital stay and faster recovery to daily life. In fact, in our cohorts, patients treated with HoLEP > $70 \mathrm{cc}$ have been discharged more than 4 days earlier than those referred to open surgery. It implies a significant reduction of indirect cost $(867.20 €$ in HoLEP group vs. 2542.87€ in OP group; $\mathrm{p}<0.001$ ), due to lower hospitalization time and lower complications rates, that leads to spare $1661.05 €$ per patients, in favor of HoLEP. Indeed, considering the global cost of both procedures, HoLEP offers a net total cost saving of $1890.82 €$ per patient as compared to OP, that assumes an important economic impact in health systems. These findings are even more impressive than those reported by Salonia and colleagues (2), reporting a significant hospital net cost savings of $9.6 \%$ in favor of HoLEP as compared with OP. However, the medical salary costs (including urologist and anesthesiologist), that could increase the cost-saving difference between two techniques, has not been included in their analyses. Despite several strength, our study is not avoided from limitations. First, number of patients included in our analyses is limited and it could affect statistical strength. Second, our cohort consists of single Italian center population with BPE submitted to most common surgical procedures (namely, HoLEP, TURP and OP) recognized as standard procedures by international guidelines (17). However, we did not include patients referred to other diffused minim invasive techniques for surgical treatment of BPE. As consequence, our cohort could not be representative of the experience of other centers both in Italy and worldwide. Third, at baseline preoperative assessment of patients with BPE, we did not provide routine urodynamic study. Fourth, our cost effectiveness analysis did not include initial costs of laser equipment that may consist of main limitation to start the procedure: the initial global costs of Holmium laser and morcellator in our department was $150.000 €$ and $50.000 €$, respectively. Of note, the economic impact of HoLEP could be overestimated, since the amount of initial financial charge, that would increase global cost of procedures, was not reported. Moreover, we did not consider how many cases are needed to amortize the initial costs of laser equipment.

\section{Conclusions}

HoLEP is a safe and valuable alternative to TURP and OP. Lower indirect costs and higher direct costs within patients treated with HoLEP, imply that global costs of HoLEP are comparable to global costs of TURP, offering a cost saving of only $11.4 €$ in favor of HoLEP.

Conversely, HoLEP proved to be a strong competitor of OP because of consistent reduction of indirect cost, mainly due to lower hospitalization time, that leads to significant global cost sparing amounting to $1890.82 €$ in favor of HoLEP. However, further evaluations including 
the initial cost of laser equipment and multicentric experiences are needed to assess the real economic advantages of laser prostatectomy compared with standard surgical approaches.

\section{References}

1. DerSarkissian M, Xiao Y, Duh MS, et al. Comparing Clinical and Economic Outcomes Associated with Early Initiation of Combination Therapy of an Alpha Blocker and Dutasteride or Finasteride in Men with Benign Prostatic Hyperplasia in the United States. Journal of managed care \& specialty pharmacy. 2016; 22:1204-14.

2. Salonia A, Suardi N, Naspro R, et al. Holmium laser enucleation versus open prostatectomy for benign prostatic hyperplasia: an inpatient cost analysis. Urology. 2006; 68:302-6.

3. Cindolo L, Pirozzi L, Fanizza C, et al. Drug adherence and clinical outcomes for patients under pharmacological therapy for lower urinary tract symptoms related to benign prostatic hyperplasia: population-based cohort study. Eur Urol. 2015; 68:418-25.

4. Presicce F, C DEN, Gacci M, et al. The influence of the medical treatment of LUTS on benign prostatic hyperplasia surgery: do we operate too late? Minerva Urol Nefrol. 2017; 69:242-52.

5. Schroeck FR, Hollingsworth JM, Kaufman SR, et al. Population based trends in the surgical treatment of benign prostatic hyperplasia. J Urol. 2012; 188:1837-41.

6. Messina R, Mirone V. Benign Prostatic Hyperplasia - An economic assessment of fixed combination therapy based on a literature review. Arch Ital Urol Androl. 2015; 87:185-9.

7. Taub DA, Wei JT. The economics of benign prostatic hyperplasia and lower urinary tract symptoms in the United States. Curr Urol Rep. 2006; 7:272-81.

8. Krambeck AE, Handa SE, Lingeman JE. Experience with more than 1,000 holmium laser prostate enucleations for benign prostatic hyperplasia. J Urol. 2013; 189(1 Suppl):S141-5.

9. Tooher R, Sutherland P, Costello A, et al. A systematic review of holmium laser prostatectomy for benign prostatic hyperplasia. J Urol. 2004; 171:1773-81.

10. Lourenco T, Pickard R, Vale L, et al. Alternative approaches to endoscopic ablation for benign enlargement of the prostate: systematic review of randomised controlled trials. BMJ. 2008; 337:a449.

11. Tan A, Liao C, Mo Z, Cao Y. Meta-analysis of holmium laser enucleation versus transurethral resection of the prostate for symptomatic prostatic obstruction. Br J Surg surgery. 2007; 94:1201-8.

12. Montorsi F, Naspro R, Salonia A, et al. Holmium laser enucleation versus transurethral resection of the prostate: results from a 2 center prospective randomized trial in patients with obstructive benign prostatic hyperplasia. J Urol. 2008; 179 (5 Suppl):S87-90.

13. Kuntz RM, Ahyai S, Lehrich K, Fayad A. Transurethral holmium laser enucleation of the prostate versus transurethral electrocautery resection of the prostate: a randomized prospective trial in 200 patients. J Urol. 2004; 172:1012-6.

14. Naspro R, Suardi N, Salonia A, et al. Holmium laser enucleation of the prostate versus open prostatectomy for prostates > $70 \mathrm{~g}$ : 24month follow-up. Eur Urol. 2006; 50:563-8.

15. Kuntz RM, Lehrich K, Ahyai SA. Holmium laser enucleation of the prostate versus open prostatectomy for prostates greater than 100 grams: 5-year follow-up results of a randomised clinical trial. Eur Urol. 2008; 53:160-6.

16. Elshal AM, Mekkawy R, Laymon M, et al. Holmium laser enucleation of the prostate for treatment for large-sized benign prostate hyperplasia; is it a realistic endourologic alternative in developing country? World J Urol. 2016; 34:399-405.

17. Gratzke C, Bachmann A, Descazeaud A, et al. EAU Guidelines on the Assessment of Non-neurogenic Male Lower Urinary Tract Symptoms including Benign Prostatic Obstruction. Eur Urol. 2015; 67:1099-109.

18. Fraundorfer MR, Gilling PJ, Kennett KM, Dunton NG. Holmium laser resection of the prostate is more cost effective than transurethral resection of the prostate: results of a randomized prospective study. Urology. 2001; 57:454-8.

19. Fayad AS, Elsheikh MG, Zakaria T, et al. Holmium Laser Enucleation of the Prostate Versus Bipolar Resection of the Prostate: A Prospective Randomized Study. "Pros and Cons". Urology. 2015; 86:1037-41.

20. Mathieu R, Lebdai S, Cornu JN, et al. Perioperative and economic analysis of surgical treatments for benign prostatic hyperplasia: A study of the French committee on LUT. Prog Uro. 2017; 27:362-8.

21. Rassweiler J, Schulze M, Stock C, et al. Bipolar transurethral resection of the prostate--technical modifications and early clinical experience. Minim Invasive Ther Allied Technol. 2007; 16:11-21.

22. Gilling PJ, Kennett K, Das AK, et al. Holmium laser enucleation of the prostate (HoLEP) combined with transurethral tissue morcellation: an update on the early clinical experience. J Endourol. 1998; 12:457-9.

23. Tubaro $A$, de Nunzio $C$. The current role of open surgery in $B P H$. EAU-EBU Update Series. 2006;4:191-201.

24. Dindo D, Demartines N, Clavien PA. Classification of surgical complications: a new proposal with evaluation in a cohort of 6336 patients and results of a survey. Ann Surg. 2004; 240:205-13.

25. Cornu JN, Ahyai S, Bachmann A, et al. A systematic review and meta-analysis of functional outcomes and complications following transurethral procedures for lower urinary tract symptoms resulting from benign prostatic obstruction: an update. Eur Urol. 2015; 67:1066-96.

26. Vincent MW, Gilling PJ. HoLEP has come of age. World J Urol. 2015; 33:487-93.

\section{Correspondence}

Riccardo Schiavina, MD - rschiavina@yahoo.it

Lorenzo Bianchi, MD (Corresponding Author)

lorenzo.bianchi3@gmail.com

Marco Borghesi, MD - mark.borghesil@gmail.com

Hussam Dababneh, MD - drdababneh@gmail.com

Francesco Chessa, MD - francesco.chessa@live.it

Cristian Pultrone, MD - cristian28@libero.it

Andrea Angiolini, MD - dr.angiolini@gmail.com

Umberto Barbaresi, MD - ubarbaresi@libero.it

Matteo Cevenini, MD - matteoceve@gmail.com

Fabio Manferrari, MD - fabio.manferrari@unibo.it

Eugenio Brunocilla, MD - eugenio.brunocilla@unibo.it

Alessandro Bertaccini, MD - alessandro.bertaccini@gmail.com

Department of Urology, University of Bologna, Bologna (Italy)

Marco Giampaoli, MD - giampaoli.marco85@gmail.com

Angelo Porreca, MD - angelo.porreca@gmail.com

Department of Urology, Abano Terme Hospital, Padua (Italy) 